\title{
Non-conventional role of haemoglobin beta in breast malignancy
}

\author{
Marco Ponzetti ${ }^{1}$, Mattia Capulli ${ }^{1}$, Adriano Angelucci ${ }^{1}$, Luca Ventura ${ }^{2}$, Simona Delle Monache ${ }^{1}$, \\ Cinzia Mercurio ${ }^{2}$, Alessia Calgani ${ }^{1}$, Patrizia Sanità ${ }^{1}$, Anna Teti ${ }^{*}{ }^{1}$ and Nadia Rucci ${ }^{1}$ \\ ${ }^{1}$ Department of Biotechnological and Applied Clinical Sciences, University of L'Aquila, L'Aquila, Italy and ${ }^{2}$ Department of \\ Pathology, San Salvatore Hospital, L'Aquila, Italy
}

Background: Besides its role as oxygen transporter, recent findings suggest that haemoglobin beta (HBB) may have roles in other contexts.

Methods: We evaluated the impact of HBB expression in primary human breast cancers, and in breast cancer cell lines overexpressing HBB by in vitro and in vivo studies. Publicly available microarray databases were used to perform multivariate survival analyses.

Results: A significantly higher expression of HBB was observed in invasive carcinoma histotypes vs in situ counterparts, along with a positive correlation between HBB and the Ki67 proliferation marker. HBB-overexpressing breast cancer cells migrate and invade more, show HIF-1 $\alpha$ upregulation and their conditioned media enhances angiogenesis. Blocking the oxygen-binding site of HBB reverts the increase of migration and HIF-1 $\alpha$ upregulation observed in HBB-overexpressing breast cancer cells. Orthotopically implanted MDA-MB-231 overexpressing HBB (MDA-HBB) generated tumours with faster growth rate and increased neoangiogenesis. Moreover, local recurrence and visceral metastases were observed only in MDA-HBB-implanted mice. Similar results were observed with $4 \mathrm{~T} 1$ mouse breast cancer cells. Finally, bioinformatics analyses of public data sets correlated high HBB expression with lower overall survival.

Conclusions: HBB expression increases breast cancer cells aggressiveness and associates with poor prognosis, pointing to HBB as a novel biomarker for breast cancer progression.

Breast cancer is the most common malignant tumour and one of the most frequent causes of cancer-related death in women. As for the other types of cancer, the mechanisms underlying breast cancer tumourigenesis and metastasis are so far to be completely understood. Although the chances of remission in metastases-free breast carcinoma patients is favourable, life expectancy dramatically drops in patients with recurrences (Reed et al, 2012; Braden et al, 2013). Therefore, there is an urgent need to better understand the biology of this tumour and help identify prognostic factors and pathways that are targetable for therapy. In a previous study, we performed whole-genome microarray analysis in bone metastases from breast cancer patients who developed secondary lesions also in visceral organs, or suffered only of bone metastases (Capulli et al, 2012). Our bioinformatics elaboration unveiled a cluster of genes that differed significantly between the two groups. We noticed that a number of them was involved in oxygen binding and transport. Haemoglobin beta (HBB) belonged to this set and had never been previously associated with the metastatic phenotype of breast cancer.

$\mathrm{HBB}$ is a member of the globin family, a structurally conserved group of proteins often containing the heme group, which have the ability to reversibly bind $\mathrm{O}_{2}$ and other gaseous ligands in erythrocytes (Bonaventura et al, 2013). In vertebrates, HBB expression has been found also in many non-erythroid cells, including activated macrophages, alveolar epithelial type II cells and mesangial cells (Liu et al, 1999; Bhaskaran et al, 2005; Nishi

*Correspondence: Professor A Teti; E-mail: annamaria.teti@univaq.it

Received 7 November 2016; revised 31 May 2017; accepted 5 July 2017; published online 3 August 2017

(C) 2017 Cancer Research UK. All rights reserved 0007 - 0920/17 
et al, 2008). Recently, it has been demonstrated that more than $70 \%$ of breast cancer tissues were positive for $\mathrm{HBB}$, while no expression was found in normal breast tissue (Gorr et al, 2011; Capulli et al, 2012). The involvement of $\mathrm{HBB}$ in breast cancer aggressiveness was further proven by selecting MDA-MB-231 clones that developed bone and visceral metastases (MDA-BV) and comparing them with clones that developed only bone metastases (MDA-BO). The results showed that MDA-BV had a 10-fold upregulation in $\mathrm{HBB}$ compared to MDA-BO metastases (Capulli et al, 2012).

These intriguing results provided a rationale to study the involvement of HBB in breast cancer, and we identified a role for this protein in breast cancer progression, with a HIF- $1 \alpha$-dependent mechanism that stimulates migration, invasion and expression of angiogenic factors.

\section{MATERIALS AND METHODS}

Materials. DMEM (Dulbecco's modified Minimum Essential Medium), FBS (foetal bovine serum), penicillin, streptomycin and trypsin were from GIBCO (Uxbridge, UK). Sterile plastic ware was from Falcon Becton-Dickinson (Cowley, Oxford, UK) or Costar (Cambridge, MA, USA). Anti HBB antibody (\#sc-21757, mouse monoclonal $\mathrm{IgG}_{1}$ ) specific to detect human HBB (Suzuki et al, 2008) was from Santa Cruz Biotechnology Inc. (Heidelberg, Germany). The cMV6-AC vector containing the GFP-tagged ORF clone of the human haemoglobin beta $(H B B)$ (pCMV6-AC-GFP, Cat\# 203258) was purchased from Origene Technologies Inc. (Rockville, MD, USA). Lipofectamine and PLUS reagent were from Invitrogen (Carlsbad, CA, USA). TACS2 TdT DAB In situ Apoptosis Detection kit was purchased from Trevigen (Helgerman CT Gaithersburg, MD). Small interfering RNAs (siRNAs) and dharmafect reagent were from Dharmacon GE Healthcare (Lafayette, CO, USA). All other reagents were of the purest grade from Sigma Aldrich Co. (St Louis, MO, USA).

Animals. Procedures involving animals and their care were conducted in conformity with national and international laws and policies (European Economic Community Council Directive 86/609, OJ L 358, 1, December 12, 1987; Italian Legislative Decree 4.03.2014 n.26, Gazzetta Ufficiale della Repubblica Italiana no. 61, March 4, 2014; National Institutes of Health guide for the Care and Use of Laboratory Animals, National Institutes of Health Publication no. 85-23, 1985) and received Institutional approval by the Internal Ethical Board regulation (University of L'Aquila) headed by Prof. Edoardo Alesse (Approval date: 18 April 2012).

Human samples. Paraffin embedded human breast and prostate carcinomas, breast cancer bone and lymph node metastasis samples were obtained from the Pathology Unit, San Salvatore hospital, L'Aquila, Italy, with the informed consent of patients. The use of these paraffin-embedded samples did not need ethical approval.

Primary lesions from 57 consecutive and unselected patients undergone surgery from breast carcinoma, 14 lymph node metastases from the same patients, and 8 additional metastatic nodes were investigated. Normal structures and benign lesions enclosed in the samples were also analysed, as well as three additional cases of fibroadenoma and two of intraductal papilloma. Known prognostic factors such as grading, receptor status, Ki67 proliferation index, HER2 score, pT category, were available for each case (Supplementary Table 1).

Immunohistochemistry on human samples. Histological sections were deparaffinised, incubated with $0.07 \mathrm{M}$ citrate buffer ( $\mathrm{pH}$ 6), 15 min at $98{ }^{\circ} \mathrm{C}$, for antigen retrieval, treated with $3 \% \mathrm{H}_{2} \mathrm{O}_{2}$ and incubated $40 \mathrm{~min}$ at room temperature (RT) with the anti-HBB mouse monoclonal antibody. The staining signal was revealed using the Dako LSAB + System-HRP. Slides were counterstained with Mayer's haematoxylin. HBB expression in carcinoma was recorded as percentage of positively stained cells.

Cell lines. The human breast cancer cell lines MDA-MB-231, MCF7 and HCC1954, and the mouse breast cancer cell line 4T1 were obtained from the American Tissue Culture Collection (ATCC, Rockville, MD) and grown in DMEM supplemented with $10 \% \mathrm{FBS}, 100 \mathrm{IU} \mathrm{ml}^{-1}$ penicillin, $100 \mu \mathrm{g} \mathrm{ml}^{-1}$ streptomycin, and $2 \mathrm{~mm}$ L-glutamine, in a humidified $95 \%$ air $/ 5 \% \mathrm{CO}_{2}$ incubator at $37^{\circ} \mathrm{C}$.

Human umbilical vein endothelial cells (HUVECs) were purchased from Lonza (Lonza, Basel, Switzerland) and routinely cultured in endothelial cell growth medium EGM-2 (Lonza) in a humidified $95 \%$ air $/ 5 \% \mathrm{CO}_{2}$ incubator at $37^{\circ} \mathrm{C}$.

Transfection. MDA-MB-231, MCF7 and 4T1 cells were stably transfected with the expressing vector containing the GFP-tagged ORF clone of the human haemoglobin, beta (MDA-HBB, MCF7$\mathrm{HBB}$ and 4T1-HBB, respectively) or with the same vector without HBB (MDA-empty, MCF7-empty and 4T1-empty), using lipofectamine (MDA-MB-231) or Amaxa (MCF7 and 4T1). Expression was driven by the cytomegalovirus (CMV) strong promoter. Transfected cells were selected for resistance to geneticin (G418).

Treatment with siRNA. MDA-MB-231 or MCF7 cells were trypsinised and plated in $3.5 \mathrm{~cm}$ culture dishes. At $70 \%$ confluence, cells were treated with either siRNA for HBB (SmartPool) or with non-targeting siRNA 1 as negative control (final concentration, $100 \mathrm{nM}$ ), using the siRNA transfection reagent Dharmafect 1 and following the manufacturer's protocol.

Transfection with shRNAs. MDA-MB-231 cells were transfected with 4 commercially available plasmids (Origene Technologies Inc., Rockville, MD, USA) each encoding for a different shRNA specific for human $\mathrm{HBB}$ ( $\operatorname{shBB} \# 1,2,3,4)$, or with a non-targeting vector as control (shSCR). Cells were selected by puromycin resistance and then subjected to gene expression analysis to assess the shHBB vector that yielded the best silencing.

Cell viability assay. Viability of human and mouse breast cancer cell lines was evaluated by using the MTT method, according to the manufacturer's protocol (Sigma-Aldrich).

To quantify the effect of conditioned media (CM) from empty and $\mathrm{HBB}$ transfected tumour cells on HUVEC cell density, the colorimetric crystal violet method was employed. Cells were serum starved for $6 \mathrm{~h}$, then medium was replaced with EGM-2 medium with or without CM from MDA-empty and MDA-HBB. After $24 \mathrm{~h}$, cells were fixed with $3 \%$ formaldehyde plus $2 \%$ sucrose solution and stained with $5 \%$ crystal violet in $20 \%$ methanol. Stain was then dissolved with $0.1 \mathrm{M}$ sodium citrate $(\mathrm{pH} 4.2)$ and the relative absorbance evaluated at $595 \mathrm{~nm}$.

Migration and invasion assays. Migration was assessed by plating tumour cells onto $12 \mu \mathrm{m}$ polycarbonate filters coated with $4.5 \mu \mathrm{g} \mathrm{cm}^{-2}$ gelatine in the upper compartment of transwell chambers. After $6 \mathrm{~h}$ of incubation in the presence of NIH3T3 cellconditioned media used as chemoattractant, filters were stained with haematoxylin/eosin (Albini et al, 2004; Rucci et al, 2004). For cell invasion assay, procedure was the same as for migration except that the polycarbonate filters were coated with $25 \mu \mathrm{g} \mathrm{cm}^{-2}$ Matrigel and the experiment was carried out for $12 \mathrm{~h}$.

In vitro tube formation assay. The tube formation assay was carried out according to Vernon and Sage (1995). Fifteen well plates $\mu$-slides for angiogenesis (Ibidi $\mathrm{GmbH}$, Germany) were coated with Matrigel $(10 \mu \mathrm{l} /$ well $)$ at $37^{\circ} \mathrm{C}$ for $30 \mathrm{~min}$. HUVEC cells $\left(1.5 \times 10^{4}\right.$ per well $)$ were added to chilled pellets of Matrigel and incubated in the presence of conditioned media from HBB 
overexpressing or empty vector transfected tumour cells $(1: 3$ dilution). After $16 \mathrm{~h}$ the number of branching points per area was evaluated (Branching Index) using an Image J extension system for angiogenesis analysis.

Real time RT-PCR. RNA was extracted using TRIzol reagent then $2 \mu \mathrm{g}$ of RNA were reverse-transcribed into cDNA and the equivalent of $0.15 \mu \mathrm{g}$ of RNA were subjected to real time RT-PCR. All reactions were carried out using a SYBR green-based master mix containing ROX as reference dye (Sensimix, Bioline, London, UK). Data analyses were carried out via dedicated software (MxPro, Stratagene, La Jolla, CA, USA).

Real time PCR array. cDNA obtained from RNA isolated from MDA-empty and MDA-HBB cells was mixed with a SYBR Green master mix ( $\mathrm{RT}^{2}$ SYBR Green qPCR master mix) and then dispensed in the wells of the oxidative stress PCR Array (\#PAHM65Z; Superarray Biosciences). Wells were subjected to real-time PCR (Stratagene MX 3000), following the manufacturer's instructions. Array data were automatically analysed by the dedicated software, $\mathrm{RT}^{2}$ Profiler PCR Array data analysis template v3.2 (Superarray Biosciences). Briefly, each array was normalized by shifting the global minimum value to 0 to reduce the differences in fluorescence intensity in different arrays. The gene expression profiles from each array were then normalized $v s$ a set of six housekeeping genes.

Western blot analysis. For protein extraction, tumour cell lines and human erythrocytes were lysed in RIPA buffer containing protease inhibitors. Proteins were resolved by $12 \%$ (MDA-empty and MDA-HBB lysates) or 15\% (erythrocyte lysate) SDS-PAGE and transferred to nitrocellulose membranes. Blots were probed with the primary antibody for $1 \mathrm{~h}$ at room temperature, washed and incubated with the appropriate HRP-conjugated secondary antibodies for $1 \mathrm{~h}$ at room temperature. For erythrocyte protein lysates, immunoblotting with the anti-HBB antibody was performed in the absence or presence of increasing amounts of supernatant arising from hypotonic haemolysis $(0.2 \% \mathrm{NaCl}$ for $2 \mathrm{~min}$ ) of human erythrocytes. Protein bands were revealed by ECL detection.

Orthotopic tumour cell injection. Four week-old female Balb/c ${ }^{n u / n u}$ mice were anesthetized with ketamine/xylazine $\left(75 \mathrm{mg} \mathrm{kg}^{-\mathrm{g}}+\right.$ $15 \mathrm{mg} \mathrm{kg}^{-\mathrm{g}}$, respectively) by intraperitoneal injection, and MDAMB-231 or 4T1 cells stably transfected with HBB, shHBB or with an empty or non-targeting (shSCR) vector were injected $\left(1 \times 10^{6}\right.$ per50 $\mu \mathrm{l}$ PBS) into the mammary fat pad of the inguinal left breast using a tuberculin syringe with a $271 \frac{1}{2} \mathrm{G}$ needle (number of animals per group =5). After tumours became apparent we started evaluating growth over time (twice a week) by measuring their three axes $(a, b, c)$ using a calliper and applying the formula of the volume $(\mathrm{V})$ of an ellipsoid $\left(V=\frac{4}{3} \pi \mathrm{abc}\right)$. To calculate in vivo tumour growth rate data are expressed as fold increase from the first time-point.

At the 16th (MDA-MB-231) or 13th (4T1) day from injection, mice were subjected to surgical resection of the tumour, then they were monitored for two further months to evaluate any local recurrence or relapse in other organs (Rucci et al, 2013).

Evaluation of metastases. Metastases in visceral organs were detected by subjecting sacrificed mice to autopsy. All the main organs (lungs, liver, brain, spleen, gut, stomach, kidneys, bladder, and heart) were examined and mice that had clear macroscopic relapse in any of these organs were considered positive for metastasis.

Tumour histology and immunohistochemistry. Excised orthotopic tumours were fixed in $4 \%$ formaldehyde in $0.1 \mathrm{M}$ phosphate buffer, $\mathrm{pH}$ 7.2, and embedded in paraffin. Sections were cut using a Leica RM2125RT microtome. Slide-mounted tissue sections ( $4 \mu \mathrm{m}$ thick) were deparaffinised in xylene, hydrated serially in $100 \%$, $95 \%$, and $80 \%$ ethanol and stained with haematoxylin/eosin. For immunohistochemistry, sections were deparaffinised, incubated with $0.07 \mathrm{M}$ citrate buffer $(\mathrm{pH} 6), 15 \mathrm{~min}$ at $98^{\circ} \mathrm{C}$, for antigen retrieval and treated with $3 \% \mathrm{H}_{2} \mathrm{O}_{2}$ in $\mathrm{PBS}$ for $1 \mathrm{~h}$ to quench endogenous peroxidases. They were then incubated with the antiKi67, anti-CD31or anti HIF- $1 \alpha$ primary antibody for $1 \mathrm{~h}$ at room temperature. Finally, after washing, antibody binding was revealed using the Dako En-Vision Detection kit and 3,3'-Diaminobenzidine (DAB).

Evaluation of fibrotic tissue inside the tumour. Orthotopic tumour sections were stained with Masson's trichrome, observed by light microscopy and pictures were taken and analysed for the quantification of fibrotic tissue, which was carried out using ImageJ software by an unaware operator. Briefly, fibrotic tissue, stained in blue, was manually selected and the fibrotic area was measured and expressed as percent of fibrotic area over the total area per field. After evaluation, we produced binary images where the fibrotic tissue is marked in black and the rest of the tissue area is in white.

TUNEL assay for in situ apoptosis detection. Orthotopic tumour sections ( $5 \mu \mathrm{m}$ thick) were deparaffinised in xylene and hydrated serially in $100 \%, 95 \%$, and $70 \%$ ethanol. Endogenous peroxidases were quenched in $3 \% \mathrm{H}_{2} \mathrm{O}_{2}$ in methanol for $5 \mathrm{~min}$. Biotinylated nucleotides were bound to fragmented DNA by terminal deoxynucleotidyl transferase (TdT) enzyme. The labelled fragmented DNA was then detected using a Streptavidin-Horseradish Peroxidase (HRP) complex and detection was carried out using DAB. After methyl green counterstaining and dehydration, sections were mounted and analysed.

Survival analyses by publicly available microarrays. KaplanMeier survival analyses, and gene expression per risk group analysis were carried out using online-available databases (http:// bioinformatica.mty.itesm.mx:8080/Biomatec/SurvivaX.jsp, code \#GSE7390, GSE10645, and GPL5858) according to the website's instructions (Aguirre-Gamboa et al, 2013).

Statistics. Statistical analyses were performed by the unpaired Student's $t$-test, by calculating the area under the curve in multivariate data measurements, or by the analysis of variance (ANOVA) test. For Kaplan-Meier survival analyses, the log-rank test was used. Correlation studies were performed using the Spearman's correlation test. A $P$-value $<0.05$ was considered statistically significant. Statistical methods are indicated in the Figure legends.

\section{RESULTS}

HBB expression in human breast carcinoma and lymph node metastases. Immunohistochemistry for $\mathrm{HBB}$ was performed in histological sections from samples of breast carcinomas, lymph node metastases from the same patients, additional metastatic nodes and benign breast lesions, as reported in Supplementary Table 1, which includes also the clinical features. Positive staining was detected in our internal positive controls, the erythrocytes present in the sections (Figure 1A). Western blot analysis on human erythrocyte lysates confirmed that the immune-positivity was reduced when incubation with the primary antibody (anti$\mathrm{HBB}$ ) was performed in the presence of lysed erythrocyte supernatant (Supplementary Figure 1A, right panel). Along with the result shown in Figure 7B (reduced signal in HBB-knocked down cells) (see below), this observation confirmed the specificity of the HBB antibody employed in the study.

HBB positive primary tumours were 17 over 27 (63\%) poorly differentiated (G3) invasive ductal carcinomas, 6 over $13(46 \%)$ moderately differentiated (G2) ductal carcinoma and 10 over 17 
A

Infiltrating ductal carcinomas
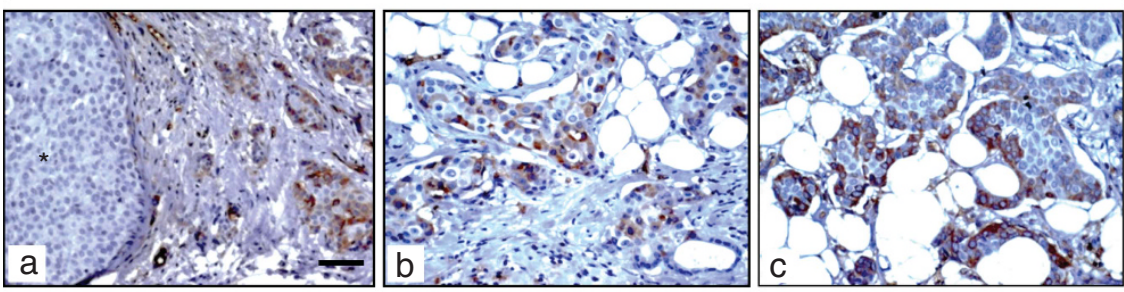

Invasive lobular $\mathrm{Ca}$

Fibroadenoma

Normal
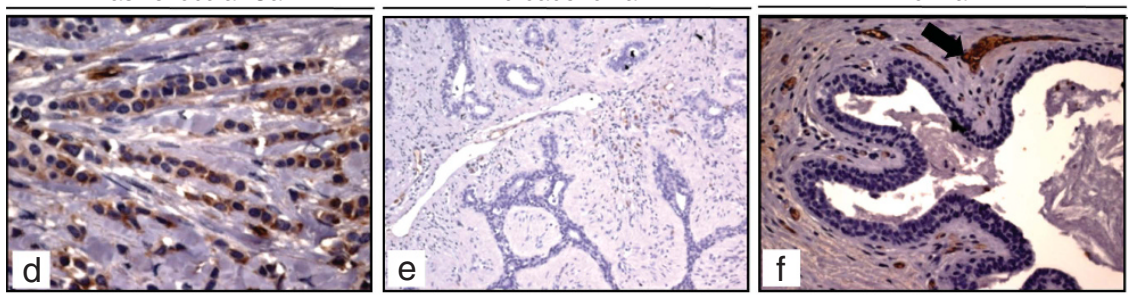

Lymph node metastases
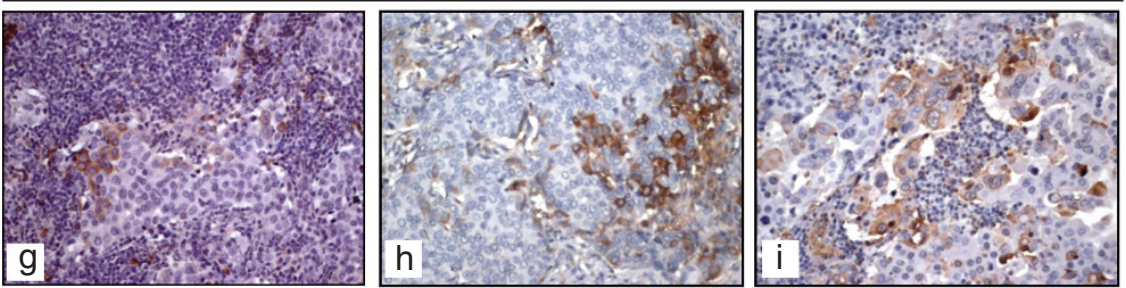

B

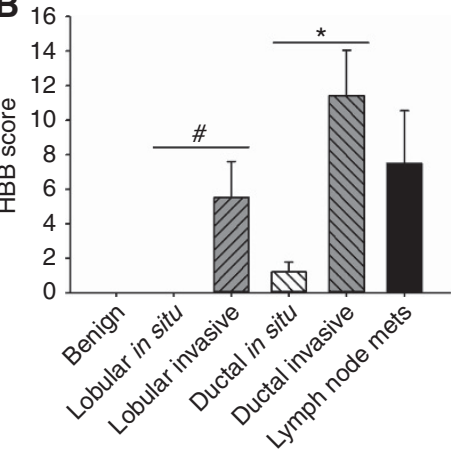

E

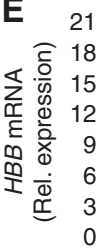

C

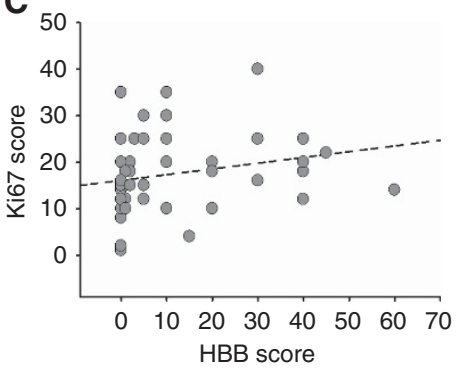

D

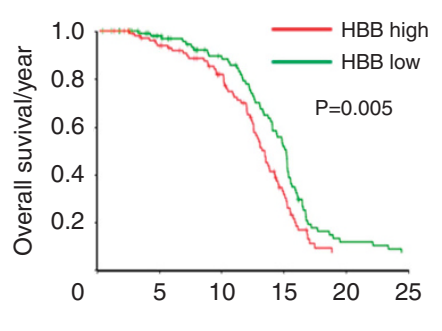

$\mathbf{F}$

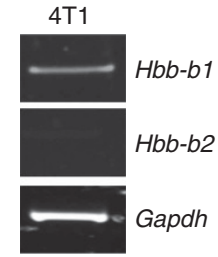

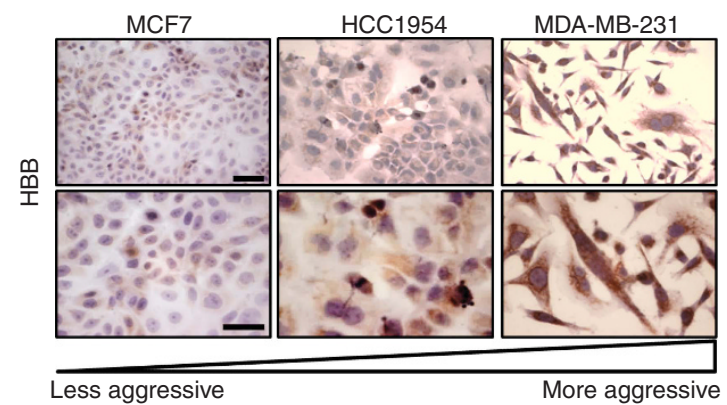

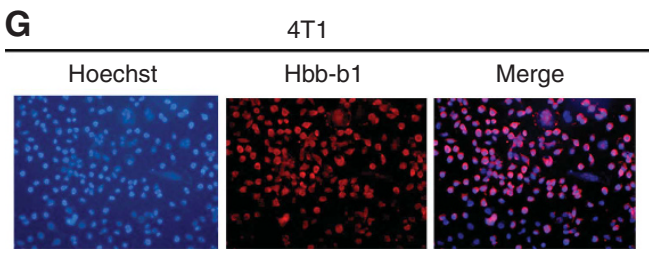

Figure 1. HBB expression in human normal and pathological samples. (A) Representative images of immunohistochemistry for HBB in human (a-d) breast carcinoma primary tumours (e) fibroadenoma, (f) normal mammary gland sample and (g-i) lymph node metastases (asterisk = in situ tumour growth). (B) HBB score in primary breast tumours, lymph node metastases from breast cancer and benign breast lesions. (C) Spearman's correlation test. (D) Kaplan-Meier curve showing the survival between patients with high or low HBB expression in primary tumours $(n=1901$, log-rank test).

(E) Transcriptional and protein expression of HBB in the indicated human breast cancer cell lines by comparative real time RT-PCR (upper panel) and immunohistochemistry (lower panels, Bar $=40 \mu \mathrm{m}$ ), respectively. (F) Transcriptional and (G) protein expression of Hbb in the mouse breast cancer cell line 4T1 by RT-PCR and immunofluorescence, respectively. In (B) ${ }^{*} P=0.022$ and ${ }^{*} P=0.003$, unpaired Student's $t$-test. Bar $=50 \mu \mathrm{m}$; in ( $E$ ) $n=4$ biological replicates, with 3 technical replicates, ${ }^{*} P<0.05$ vs HCC1954 and MCF7, one way ANOVA. $(\mathbf{F}, \mathbf{G}) n=3$ biological replicates, with 3 technical replicates. 
(59\%) invasive lobular carcinoma. Percentage of HBB positive tumour cells ranged from $1 \%$ to $45 \%$ of the total number of tumour cells (Supplementary Figure 1B). Benign lesions tested, such as fibroadenoma and papilloma, as well as adenosis, papillomatosis and normal tissue observed outside the main tumour nodule, were all $\mathrm{HBB}$-negative (Figure 1A and B). Eight lymph node metastases out of $22(36 \%)$ were positive for $\mathrm{HBB}$ (Figure 1A and B).

By means of statistical analysis of HBB score in tumour tissue, we found that in the invasive ductal carcinoma histotype, tumour cells presented with a significantly higher expression of $\mathrm{HBB}$ compared to those of the in situ counterpart (Figure 1B). A higher expression of $\mathrm{HBB}$ in ductal invasive carcinoma was also observed vs lobular invasive histotype, while the lobular in situ counterpart was negative for $\mathrm{HBB}$. No significant differences of $\mathrm{HBB}$ score were found between primary tumours and lymph node metastases (Figure 1B). Interestingly, we found a positive correlation between $\mathrm{HBB}$ expression and the Ki67 proliferation marker (Figure 1C) but not with ER, PR, and HER2 expression (Supplementary Figure 2A-C).

We next addressed the impact of $\mathrm{HBB}$ expression on breast cancer in large cohorts of patients $(n=1901)$ by using publicly available microarray databases (SurvExpress meta-analysis), observing that the overall survival was significantly lower in patients with a higher HBB expression in the primary tumour (Figure 1D). This correlation was even stronger considering only more aggressive subtypes of breast cancer: ER negative vs ER positive tumours (Supplementary Figure 3A and B), high grading vs low grading (Supplementary Figure $3 \mathrm{C}-\mathrm{E}$ ) and in more malignant $v s$ less malignant subtypes (Supplementary Figure 3F-J).

On the basis of these observations and given the similarities between breast and prostate cancer, we wondered whether the latter could also have an advantage by expressing HBB. Indeed, a weak and focal cytoplasmic positivity was observed only in 1 out of 22 prostate adenocarcinomas, while the neoplastic cells in the remaining tumours and lymph node metastases were negative (Supplementary Figure 4A). Consistent with our findings, transcriptional expression of $\mathrm{HBB}$, when present, was not associated with changes in the survival of prostate cancer patients (Supplementary Figure 4B).

In agreement with the data gathered so far, the transcriptional and protein expression of $\mathrm{HBB}$ was lower in poorly aggressive human breast cancer cell lines (MCF7, HCC1954) when compared to highly aggressive breast cancer cells (MDA-MB-231) (Figure 1E). Also the mouse high-grading breast cancer cell line, 4T1, was HBB-positive (Figure $1 \mathrm{~F}$ and $\mathrm{G}$ ). Taken together, these data strongly suggest that $\mathrm{HBB}$ expression could be associated with a more aggressive phenotype of breast cancer cells.

HBB overexpression increases tumour invasiveness. To deeply investigate the role of $\mathrm{HBB}$ in tumour progression and unveil whether the phenotype of tumour cells could be influenced by the increase in $\mathrm{HBB}$ expression, we stably overexpressed $\mathrm{HBB}$ in breast cancer cell lines (MDA/MCF7/4T1-HBB, Figure 2A, and Supplementary Figure 5A). Overexpression of $\mathrm{HBB}$ did not affect tumour cell adhesion (Figure $2 \mathrm{~B}$ and $\mathrm{C}$ ), while survival rate was unremarkable in MDA-HBB cells (Figure 2D) and increased in 4T1-HBB compared to cells transfected with empty vector (Figure 2E). Interestingly, in both MDA and 4T1 overexpressing $\mathrm{HBB}$ we found a significant increase of motility (Figure 2F) and ability to migrate and invade through gelatine and matrigel, respectively (Figure $2 \mathrm{G}$ and $\mathrm{H}$ ). This was associated with a significant increase of metalloproteinase (MMP)-9 expression at transcriptional (Figure 2I) and protein (Figure 2J) level. Consistently, knocking down $\mathrm{HBB}$ in MDA-MB-231 with siRNA (Figure 3A) did not affect neither survival (Figure 3B) nor proliferation (Figure 3C), while it significantly reduced migration, invasion and $M M P-9$ mRNA expression, compared to non-targeting siRNA-treated cells (Figure 3D-F), thus complementing the results obtained with MDA-HBB overexpressing cells.

Intriguingly, the poorly aggressive human MCF7-HBB cells showed a significant increase of survival (Supplementary Figure 5B) and migration (Supplementary Figure 5C), while invasion and MMP-9 expression were unaffected (Supplementary Figure 5D and E). Moreover, in these HBB overexpressing cells, vimentin and $\alpha$-SMA mesenchymal markers were significantly increased, while ZEB1, $\beta$-catenin and the epithelial marker E-cadherin were unchanged (Supplementary Figure 5F). Intriguingly, knocking down HBB in MCF7-HBB cells by using siRNAs specific for HBB (Supplementary Figure $5 \mathrm{G}$ ) was able to revert the increase of both $\alpha$-SMA (Supplementary Figure $5 \mathrm{H}$ ) and vimentin (Supplementary Figure 5I).

HBB overexpression induces tumour-mediated angiogenesis. Another hallmark of tumour progression is the ability of tumour cells to induce neoangiogenesis, providing nutrients and a route for tumour metastasis. Of note, MDA-HBB cells expressed higher transcriptional levels of $V E G F$ than the MDA-empty (Figure 4A), while HBB knock down reduced VEGF mRNA (Figure 4B). Consistently, conditioned medium from MDA-HBB cells significantly stimulated in vitro HUVEC tube formation (Figure 4C) and increased cell density (Figure 4D). Similarly, HBB overexpression in 4T1 cells increased Vegf transcriptional expression (Figure 4E) and in vitro tube formation of HUVEC (Figure 4F), although there was no effect on cell density (Figure 4G). In contrast, MCF7-HBB did not trigger angiogenesis (Supplementary Figure 6A) and, consistently, VEGF mRNA expression was unaffected (Supplementary Figure 6B).

Finally, we cultured MDA-empty and MDA-HBB cells for $72 \mathrm{~h}$ at extreme hypoxia $\left(0.1 \% \mathrm{O}_{2}\right.$ humidified atmosphere), but we did not find any difference in terms of fold endpoint cell number between the two cell lines (Supplementary Figure 7A). Moreover, under these conditions $\mathrm{HBB}$ basal levels did not change in MDA cells (Supplementary Figure 7B) while in the MCF7, which usually do not express HBB, its mRNA expression was induced by hypoxia (Supplementary Figure 7C), thus suggesting a role for this protein in the response to oxygen reduction. Finally, an opposite result was observed in 4T1 cells, in which $\mathrm{HBB}$ mRNA was reduced (Supplementary Figure 7D). Taken together, these data suggest different pathways of regulation of $\mathrm{HBB}$ under hypoxia, likely reflecting the heterogeneity of these breast cancer cell types.

The oxygen binding capacity of HBB mediates its function in tumour cells. To gain some insights into the mechanism of action of HBB in our cell models, we investigated the role of HBB in oxidative stress handling by performing a real time array, which included 84 genes involved in this function (Supplementary Figure $8 \mathrm{~A}$ and $\mathrm{B}$ ), confirming the regulation of the most relevant genes by real time RT-PCR (Figure 5A). The hypoxia inducible factor- $1 \alpha$ (HIF- $1 \alpha)$, a key regulator of cancer cell aggressiveness, was significantly reduced in MDA cells treated with HBB-specific siRNA, both at the mRNA and protein level (Figure 5B), while the hydroxylated form of HIF- $1 \alpha$ (OH-HIF- $1 \alpha)$ was not affected by HBB knock down (Figure 5C).

Bioinformatics analysis performed using the web-based software for protein-protein interaction STRING v9.1 (Franceschini et al, 2013) revealed a common interactive pathway for HIF- $1 \alpha$, VEGF, Nitric-Oxide Synthase 2 (NOS2) and MMP-9 (Supplementary Figure $8 \mathrm{C}$ ). Other genes observed to be upregulated by $\mathrm{HBB}$ overexpression were functionally linked together, such as dual specificity phosphatase 1 (DUSP1) and NOS2, both being upregulated in breast cancer cells during hypoxia (Lu et al, 2006; Chiarugi et al, 1999), while phosphatidylinositol-3,4,5-trisphosphate-dependent Rac exchange factor 1 (PREX1) was upregulated 
A

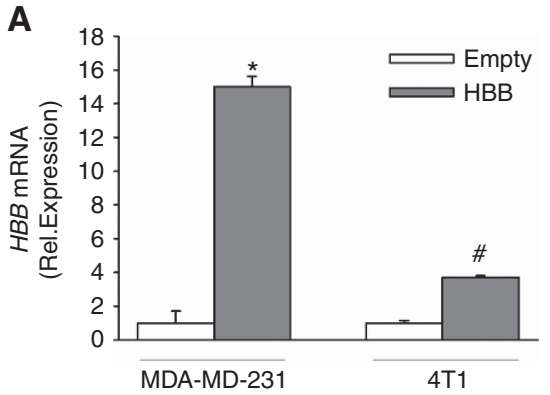

C

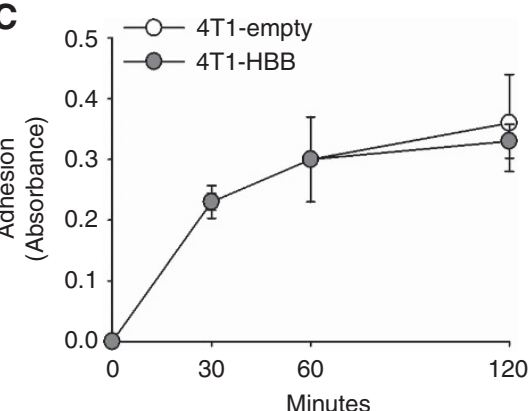

E
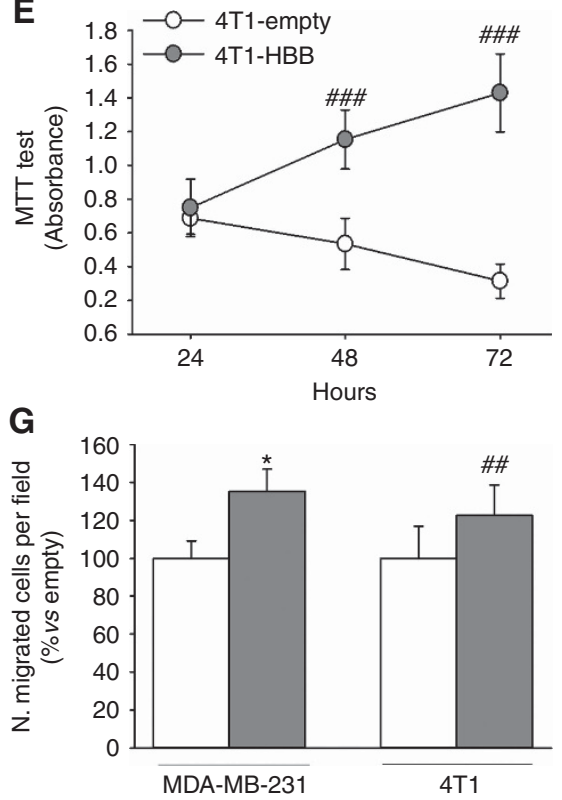

I

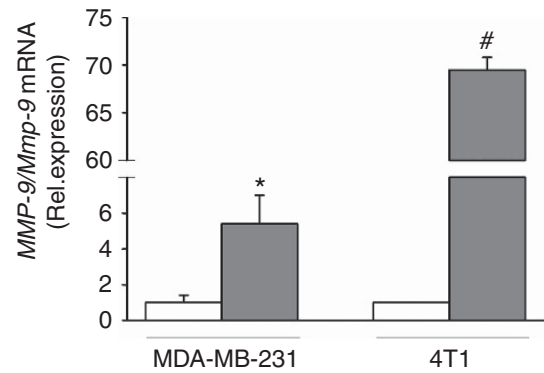

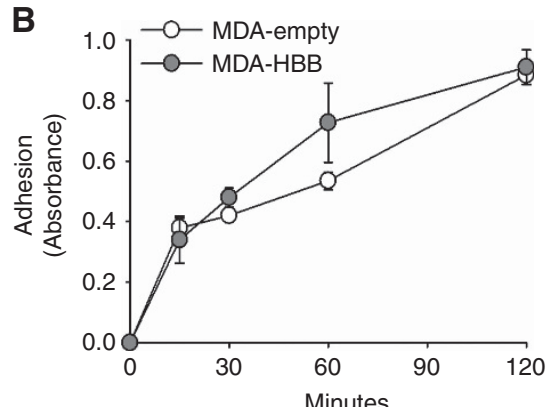

D
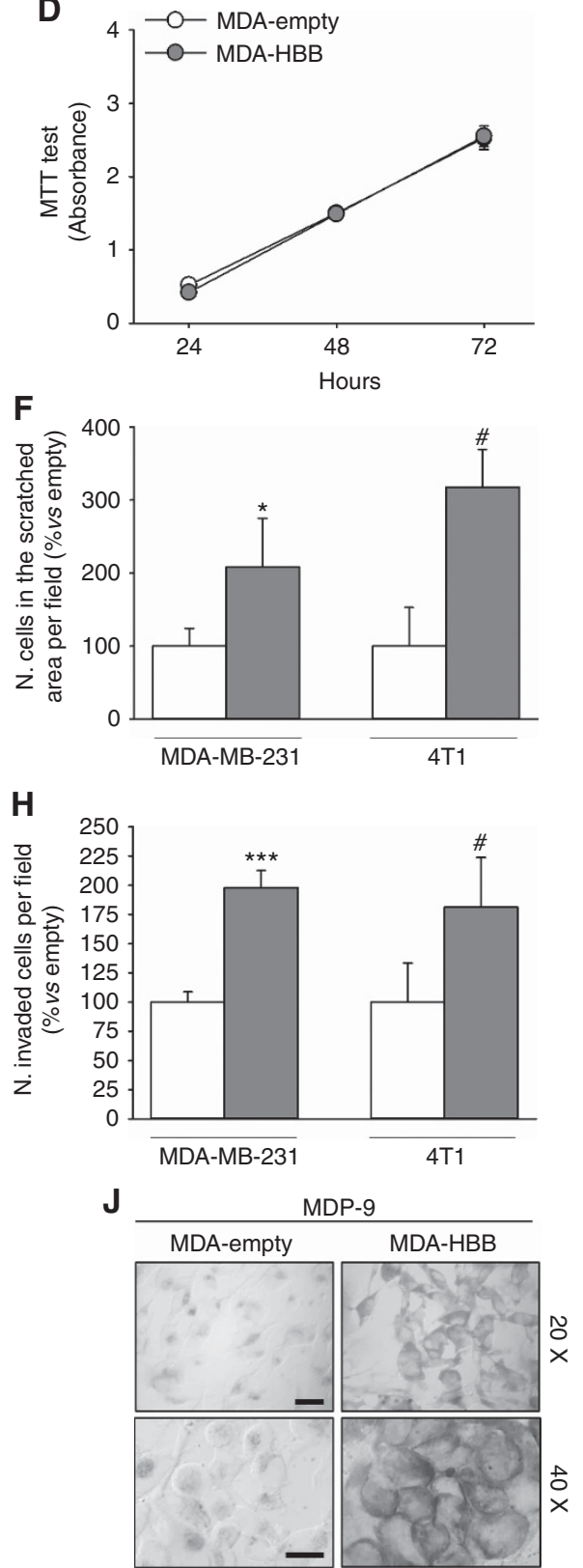

Figure 2. Effect of HBB overexpression on breast cancer cell phenotype. The human breast cancer cell line MDA-MB-231 and the mouse breast cancer cell line 4T1 were stably transfected with a plasmid carrying HBB (MDA-HBB and 4T1-HBB) or with an empty vector as control (MDA-empty and 4T1-empty). (A) Transcriptional expression of HBB by comparative real time RT-PCR. Evaluation of (B) MDA-empty and -HBB or (C) 4T1-empty and -HBB adhesion on FBS coated wells, (D) MDA-empty and -HBB or (E) 4T1-empty and -HBB growth rate by MTT test. Evaluation of (F) motility by wound healing assay, $(\mathbf{G})$ migration and $(\mathbf{H})$ invasion assay across gelatine and matrigel coated transwells, respectively. (I) Transcriptional expression of MMP-9 evaluated by comparative real time RT-PCR. (J) Immunocytochemistry of MMP-9 expressed on plasma membrane. Results are $(\mathbf{A}-\mathbf{I})$ the mean \pm s.d. or $(\mathbf{J})$ representative of $n=3$ biological replicates with at least 3 technical replicates each $\left({ }^{\star} P<0.05\right.$, and ${ }^{\star * \star} P=1 \times 10^{-4}$ vs MDA-empty; ${ }^{\#}<0.05,{ }^{\# \#} P<0.005$ and ${ }^{\# \# \#} P<1 \times 10^{-4}$ vs 4 T1-empty unpaired Student's t-test). Bar $=15 \mu \mathrm{m}$. 
A
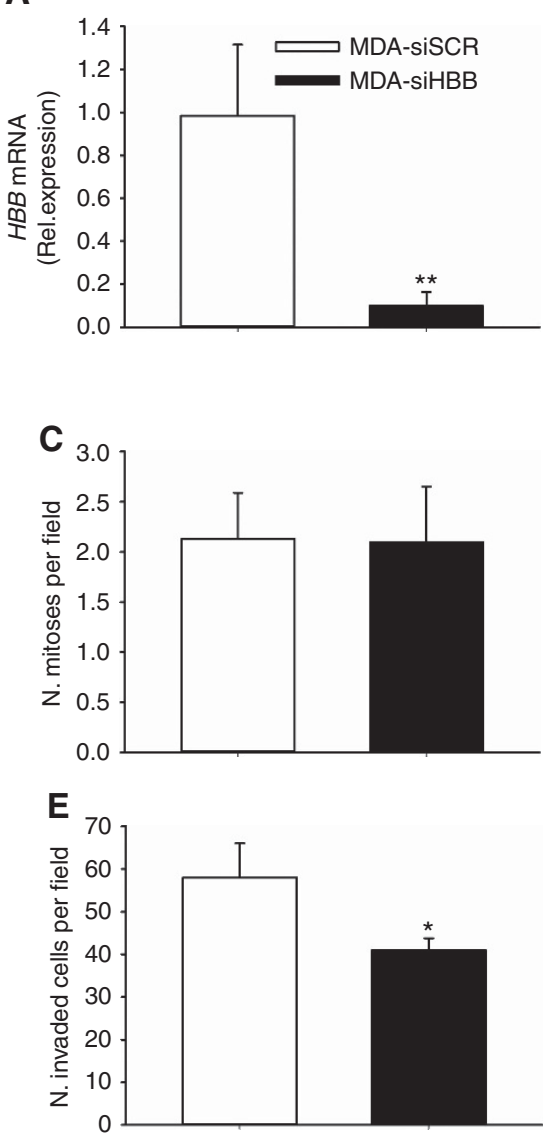
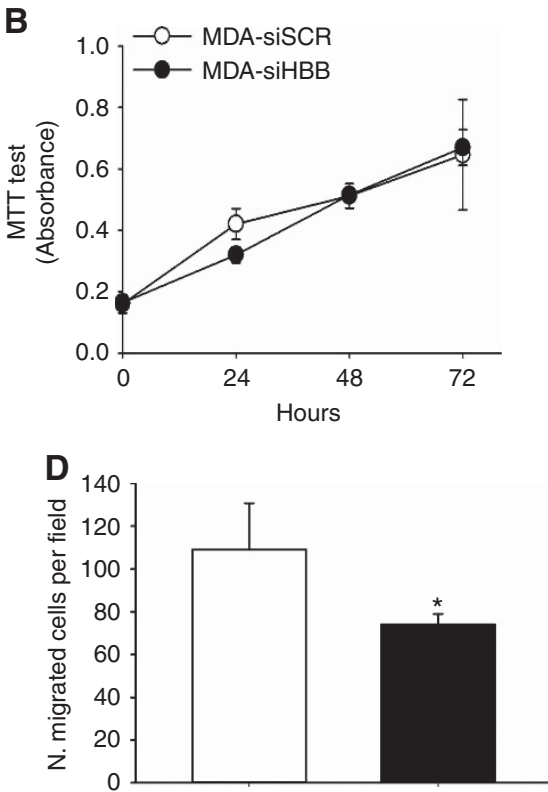

$\mathbf{F}$

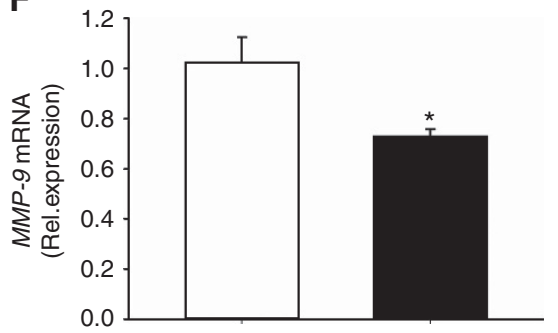

Figure 3. Effect of HBB knock down on human breast cancer cell phenotype. The human breast cancer cell line MDA-MB-231 was subjected to RNA silencing using siRNA specific for HBB (MDA-siHBB) or to treatment with a non-targeting scrambled siRNA (MDA-siSCR) as control.

(A) Transcriptional expression of HBB and evaluation of (B) cell viability by MTT test, (C) proliferation by counting the number of mitosis after cell staining with Hoechst, (D) migration and (E) invasion across gelatine and matrigel coated transwells, respectively. (F) Transcriptional expression of MMP-9. Data are the mean \pm s.d. of $n=3$ biological replicates with technical replicates $\left({ }^{\star} P<0.05\right.$ and ${ }^{\star *} P=0.002$ vs MDA-siSCR, unpaired

Student's t-test).

in response to PI3K activation, a central event in the response of tumour cells to hypoxia (Courtnay et al, 2015).

Interestingly, we found HIF-1 $\alpha$ protein basal levels to be high in the most aggressive MDA-MB-231 and 4T1 cells, while no expression was observed in MCF7 cells (Supplementary Figure $8 \mathrm{D}$ ). To strengthen the involvement of HIF- $1 \alpha$ in this context, we treated MDA-empty and -HBB transfected cells with the HIF- $1 \alpha$ inhibitor chetomin, which significantly reduced HIF- $1 \alpha$ transcriptional expression (Supplementary Figure 8E). Consistently, this treatment significantly reduced $M M P-9$ mRNA in both MDA-empty and MDA-HBB cells (Supplementary Figure 8F) while VEGF expression was significantly reduced only in MDAempty cells, thus suggesting that the increase of this factor in MDA-HBB is not only dependent on HIF- $1 \alpha$ signalling (Supplementary Figure 8G). Finally, wound healing assay demonstrated the ability of chetomin to revert the increase of motility observed in MDA-HBB cells (Supplementary Figure 8H).

We next analysed whether the effect of $\mathrm{HBB}$ depended on its oxygen binding function. We treated tumour cells with carbon monoxide (CO)-releasing molecule-2 (CORM-2), an inhibitor of the heme binding to oxygen, and tested its ability to prevent the effects mediated by HBB. To exclude any effect of CORM- 2 on cell viability, we first performed an MTT test, finding that this treatment did not affect survival of MDA and 4T1 cells (Supplementary Figure 9A and B, respectively). Interestingly, treatment with CORM-2 prevented the increase of motility
(Figure 5D), as well as the transcriptional (Figure 5E) and the protein (Figure $5 \mathrm{~F}$ ) upregulation of $\mathrm{HIF}-1 \alpha$ mediated by $\mathrm{HBB}$ overexpression.

Upregulation of Nos 2 and Hif- $1 \alpha$ were also found in $4 \mathrm{~T} 1-\mathrm{HBB}$ cells (Figure 5G), along with an increased motility impaired by the treatment with CORM-2 (Figure $5 \mathrm{H}$ ). In contrast, neither HIF-1 $\alpha$ nor NOS2 mRNA were changed in MCF7-HBB cells (Supplementary Figure 6C and D).

Effect of $\mathrm{HBB}$ on in vivo tumour growth. To evaluate the potential HBB-mediated effects on primary breast tumour growth and on its ability to metastasize, we performed an orthotopic injection of MDA-empty and MDA-HBB cells in female Balb/c $\mathrm{c}^{n u / n u}$ immunocompromised mice. Figure $6 \mathrm{~A}$ shows that MDA-HBB cells formed faster-growing tumours, compared to MDA-empty. After 16 days from injection, primary tumours were surgically resected. Macroscopic examination confirmed the ability of MDA-HBB cells to form significantly larger and heavier tumours (Figure 6A and $\mathrm{B}$ ). Explanted tumours from MDA-HBB cells retained HBB overexpression (Supplementary Figure 10A) and showed a trend of increase in the proliferation marker Ki67 (Figure 6C) and no modulation of cell apoptosis (Figure 6D).

Noteworthy, the endothelial marker CD31 was more expressed in MDA-HBB tumours, indicating an increased vascularization (Figure 6E). MDA-HBB tumours also exhibited a trend of increase of HIF-1 $\alpha$ (Supplementary Figure 10B). Moreover, Masson's 
A

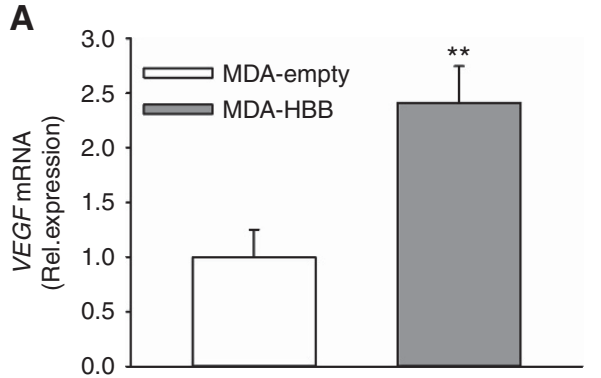

C
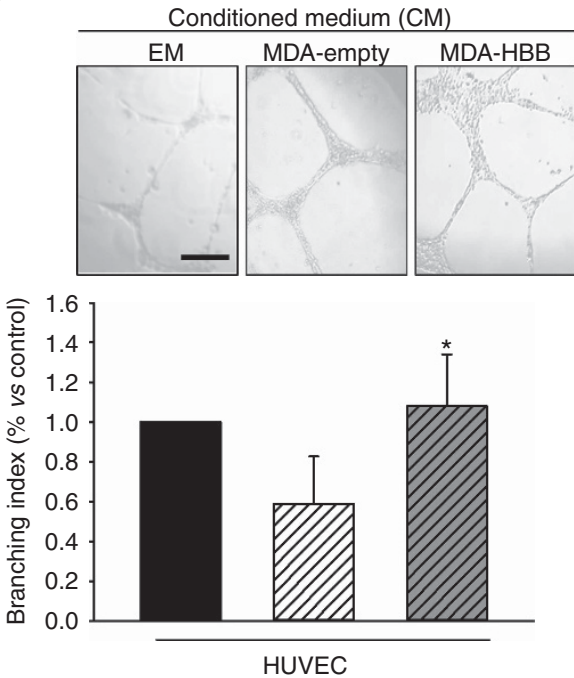

E

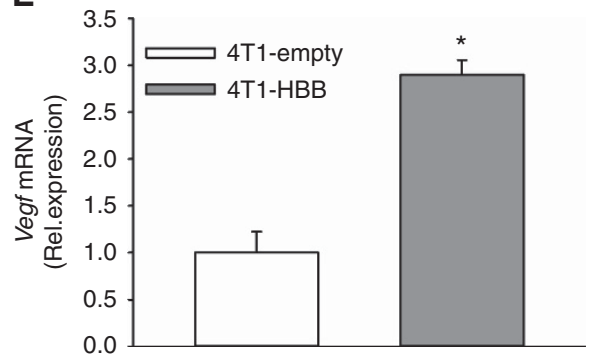

G

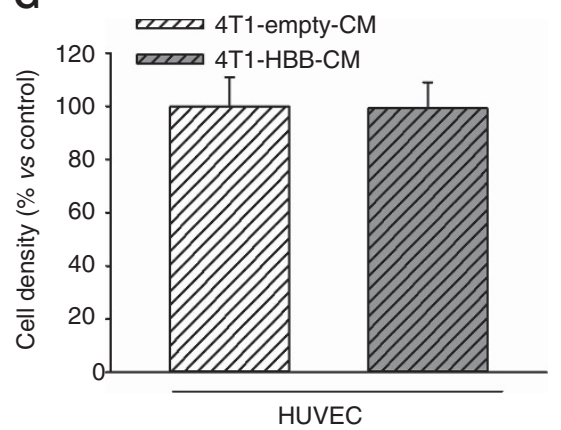

B

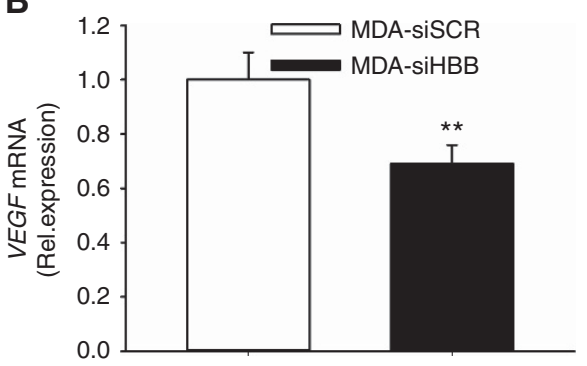

D

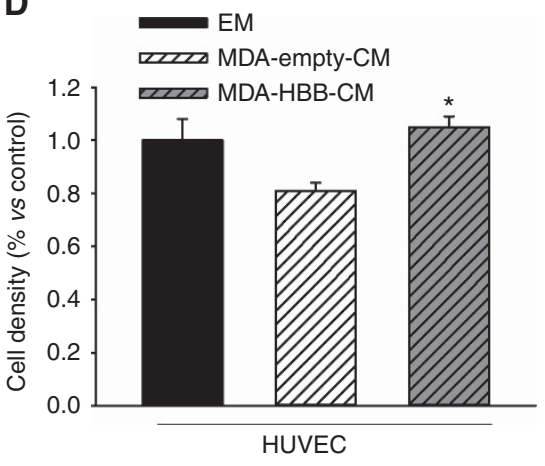

F

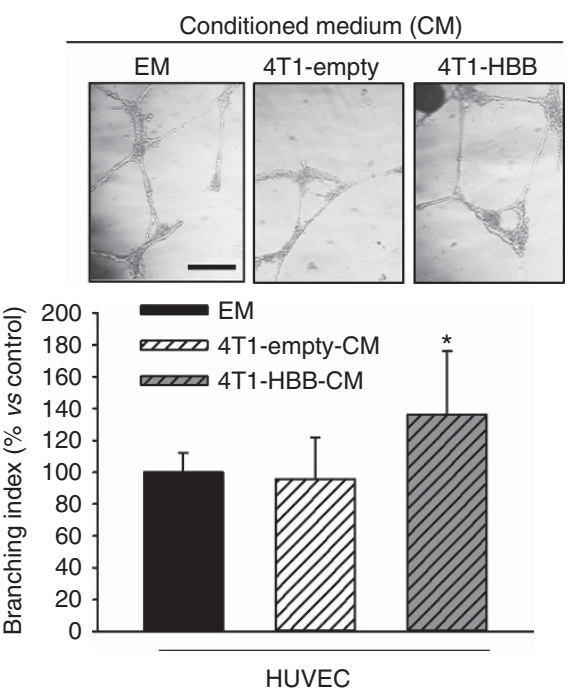

Figure 4. In vitro angiogenesis. (A,B) Transcriptional expression by real time RT-PCR of VEGF in (A) MDA-empty and MDA-HBB cells and in (B) MDA-MB-231 cells treated with siRNA specific for HBB (siHBB) or treated with a scrambled siRNA (siSCR). (C,D) Human umbilical vein endothelial cells (HUVECs) were treated for $24 \mathrm{~h}$ with endothelial medium (EM) as positive control or with conditioned medium from MDA-HBB (MDA-HBB-CM) or MDA-empty cells (MDA-empty-CM). (C) Representative images (inset) and quantification of in vitro tube formation (branching index). (D) Cell density assessed by crystal violet staining. (E) Transcriptional expression of Vegf in 4T1-empty and 4T1-HBB cells by comparative real time RT-PCR. (F,G) HUVEC cells were treated for $24 \mathrm{~h}$ with endothelial medium (EM) or with conditioned medium from 4T1-HBB (4T1-HBB$\mathrm{CM}$ ) or 4T1-empty cells (4T1-empty-CM). (F) Representative images (inset) and quantification of in vitro tube formation (branching index). (G) Cell density assessed by crystal violet staining. Data are the mean \pm s.d. of $n=3$ biological replicates with 3 technical replicates. (A-D): ${ }^{\star} P<0.05$ and ${ }^{\star}{ }^{*}=0.01$ vs MDA-empty; (E-G): ${ }^{\star} P<0.05$ vs 4 T1-empty, unpaired Student's t-test). 
A

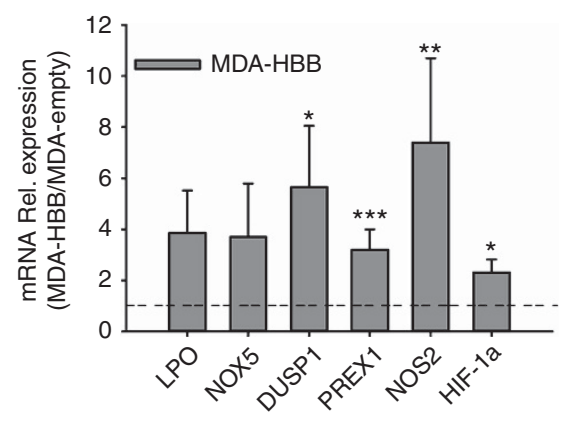

C
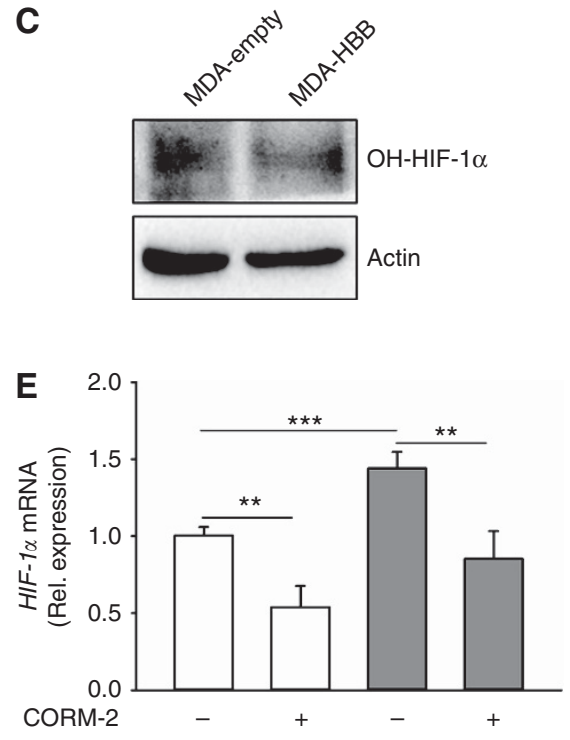

G

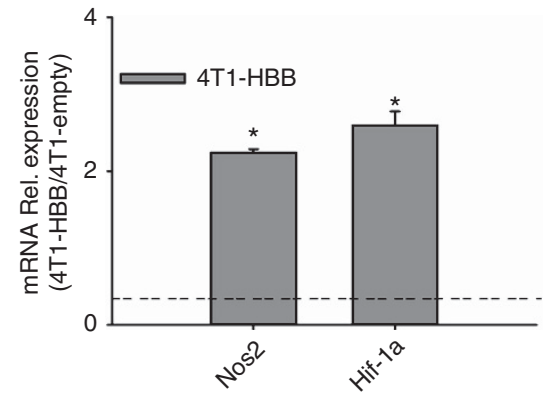

B
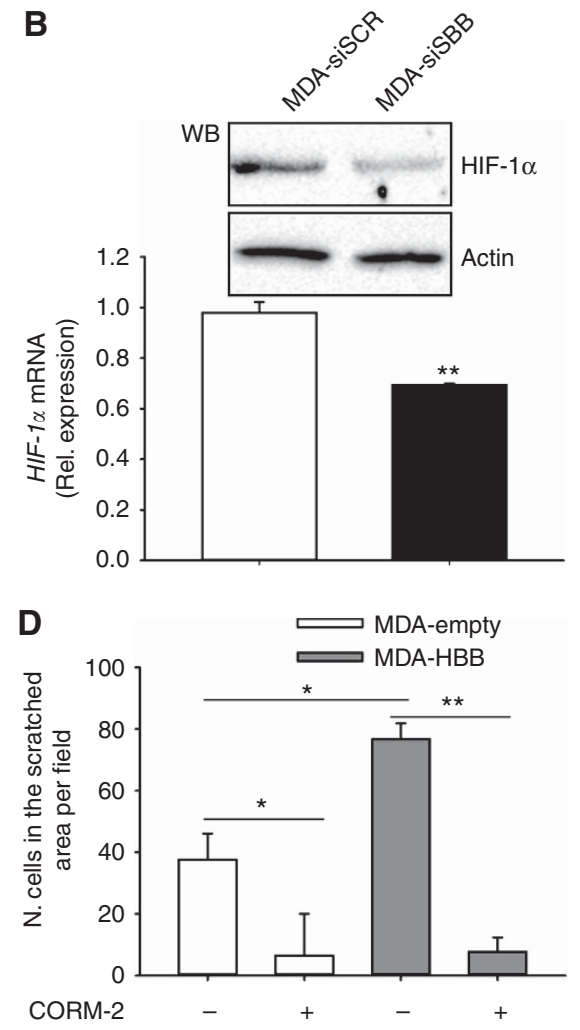

$\mathbf{F}$

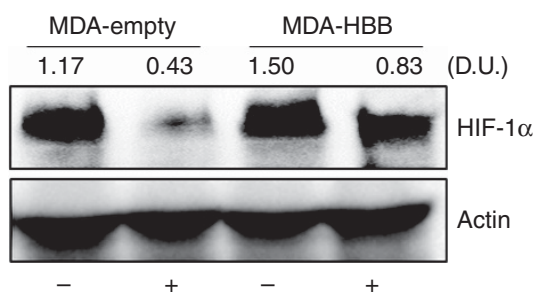

CORM-2

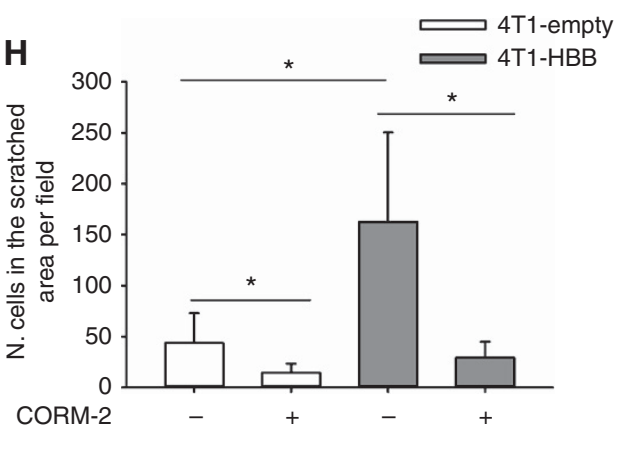

Figure 5. Mechanism of action of HBB. (A) Comparative real time RT-PCR of the indicated genes in MDA-HBB relative to MDA-empty cells (set to 1, dotted line). (B) Transcriptional (graph) and protein expression (inset) of HIF-1 $\alpha$ in MDA cells treated with siRNA specific for HBB (siHBB) or with scrambled siRNA (siSCR). (C) Protein expression of hydroxylated HIF-1 $\alpha$ (OH-HIF-1 $\alpha$ ) in MDA-empty and MDA-HBB cells. (D-F) MDA-empty and MDA-HBB were treated with vehicle or with the carbon monoxide donor CORM-2 and subjected to (D) scratch wound healing assay.

(E) Transcriptional and $(\mathbf{F})$ protein evaluation of HIF-1 $\alpha$ expression in MDA-empty and MDA-HBB cells treated with vehicle or CORM-2 (D.U. = normalized Densitometric Units). (G) Transcriptional expression of Nos2 and Hif-1 $\alpha$ in 4T1-empty and 4T1-HBB cells. (H) Scratch wound healing assay performed with 4T1-empty and 4T1-HBB cells treated with CORM-2. Data are (C, F) representative or $(A, B, D, E, G, H)$ the mean \pm s.d. of $n=3$ biological replicates with at least 3 technical replicates (A-D: ${ }^{\star} P<0.05$, ${ }^{\star \star} P<0.01$ and ${ }^{\star \star \star} P<0.005$ vs MDA-empty; F-G: ${ }^{\star \star} P<0.01$ vs 4T1-empty, unpaired Student's t-test).

trichrome staining evidenced less fibrosis in MDA-HBB tumour sections, which was confirmed by quantification of the fibrotic area over the total tumour area (Figure 6F).

Intriguingly, after 2 months from primary tumour resection, local recurrence was found in $60 \%$ of mice injected with MDAHBB cells, while recurrence was not observed in any of the mice injected with MDA-empty cells (Figure 6G). Consistently, 40\% of mice injected with MDA-HBB cells developed visceral metastases, while mice receiving MDA-empty cells did not show distant tumour relapse over the same timeframe (Figure $6 \mathrm{H}$ ).

Orthotopic injection of $4 \mathrm{~T} 1$ cells showed a greater in vivo tumour growth rate of $4 \mathrm{~T} 1-\mathrm{HBB}$ compared to $4 \mathrm{~T} 1$-empty cells 
A
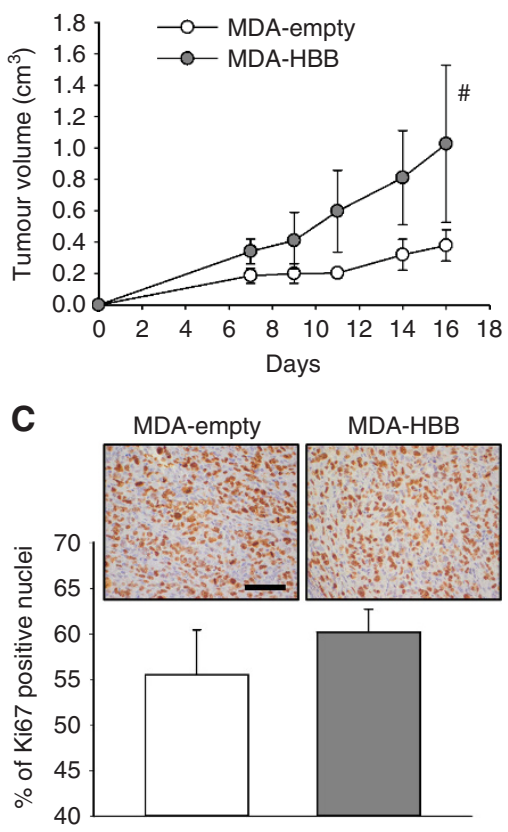

E
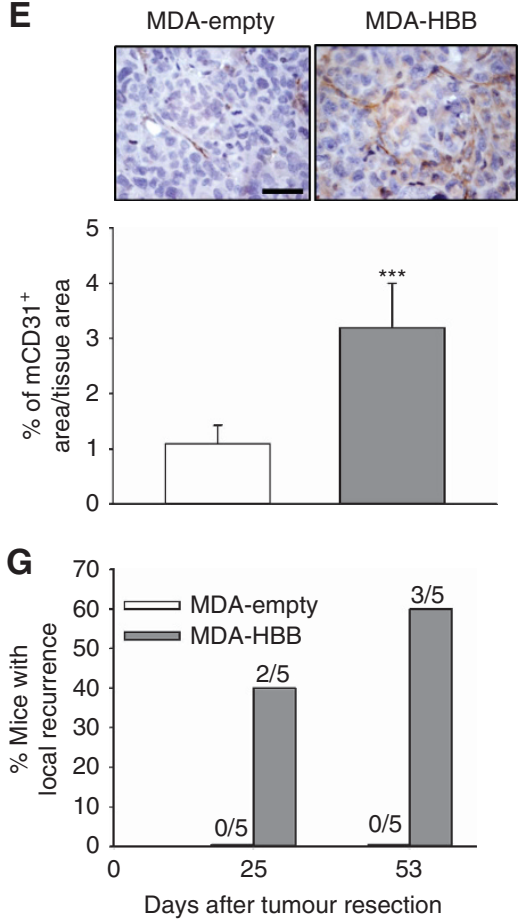

B
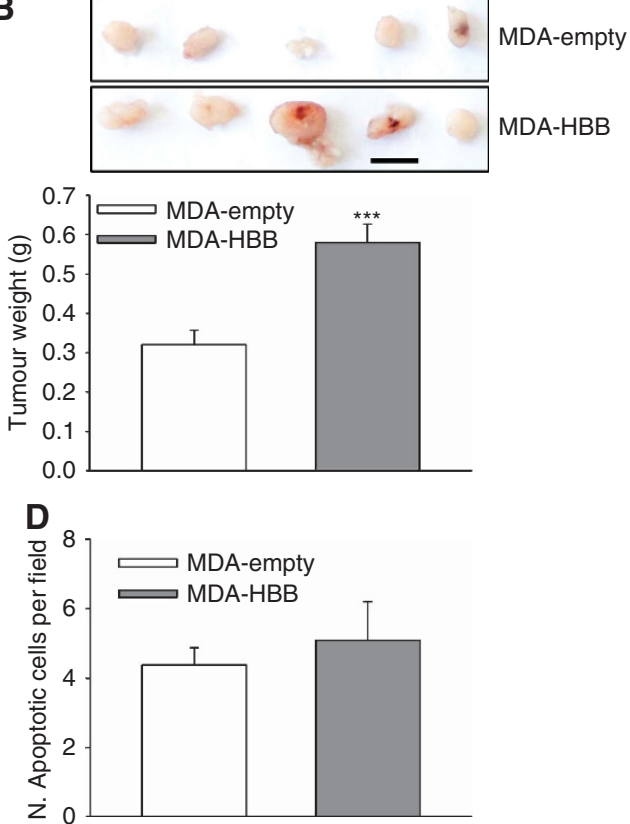

$\mathbf{F}$
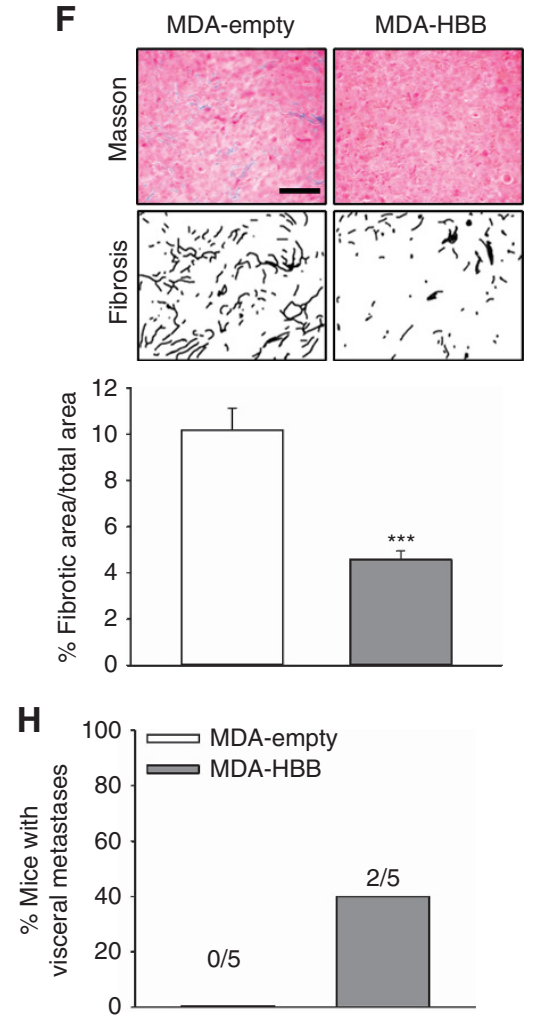

Figure 6. Effect of HBB on MDA-MB-231 orthotopic tumour growth and relapse. Four week-old Balb/cnu/nu female mice were subjected to injection of MDA-empty or MDA-HBB cells $(1 \times 106$ per $50 \mu \mathrm{l}$ PBS $)$ into the mammary fat pad. (A) Evaluation of tumour volume recorded during the timeframe of the experiment. (B) Macroscopic picture (inset) and weight of tumours surgically excised at the 16th day from injection. (C-F) Excised tumours were fixed, embedded in paraffin, cut in $5 \mu \mathrm{m}$ thin sections and subjected to (C) immunohistochemistry for Ki67, to evaluate the proliferation rate (Bar $=50 \mu \mathrm{m}$ ). (D) TUNEL assay to evaluate apoptosis. (E) Immunohistochemistry for the CD31 endothelial marker and (F) Masson's trichromic staining for quantification of fibrotic tissue (Bar $=50 \mu \mathrm{m})$. (G, H) Percentage of mice with (G) local recurrence and $(\mathbf{H})$ visceral metastases developed after tumour resection. Data are the mean \pm s.d. of $n=5$ mice per group. (C-F) Three technical replicates per sample. ( $P=0.0048$ vs MDA-empty, area under the curve; ${ }^{\star \star \star} P<0.002$ vs MDA-empty, unpaired Student's t-test).

(Supplementary Figure 11A). After 13 days, tumours were removed and histological analysis showed a higher number of mitoses in 4T1-HBB-generated tumours compared to control tumours (Supplementary Figure 11B). The CD31-positive blood vessel area did not vary significantly between the two groups (Supplementary
Figure $11 \mathrm{C}$ ), while the fibrotic area/tissue area was lower in 4T1HBB overexpressing tumours (Supplementary Figure 11D), strengthening the data on MDA-HBB cells.

To further analyse the role of HBB in tumour growth, we also performed an in vivo experiment knocking down HBB expression 
in MDA-MB-231 cells by stable transfection of shRNAs specific for HBB. Among 4 HBB shRNA sequences, we chose MDA-shHBB\#4 because it yielded the best HBB mRNA downregulation after antibiotic selection (Figure 7A), which was confirmed also at protein level (Figure 7B). Cells transfected with a non-targeting shRNA vector (shSCR) were used as control. Results showed that MDAshHBB\#4-generated tumours were significantly smaller in volume until the 16th day from tumour cells injection (Figure 7C). However, at later time-points differences between the two groups were no longer significant. Intriguingly, and consistently with the overexpression data, local recurrence 23 days after resection were found only in 1 out of $5 \mathrm{MDA}$-shHBB-injected mice, while recurrence was present in $80 \%$ mice injected with MDA-shSCR (Figure 7D).

\section{DISCUSSION}

During their progression, cancer cells can express several de novo proteins, which could represent tumour biomarkers with a real driving role in tumour progression. In our previous work, we observed that $\mathrm{HBB}$ was one of the most upregulated genes in bone metastases of a subgroup of breast cancer patients with metastases also in visceral organs compared to bone metastases from patients with metastases exclusively in bone (Capulli et al, 2012). Our aim in the present work was to understand whether HBB could be used as a biomarker of breast cancer progression, and if its expression could give breast cancer cells a selective advantage. To this aim, we analysed primary breast carcinomas, and observed a correlation between $\mathrm{HBB}$ expression and breast cancer aggressiveness. Notably, inside the same tumour, the areas with histological invasive features exhibited higher numbers of HBB positive cells compared to the in situ counterpart, while the normal mammary epithelium was HBB-negative.

Similar positive correlation between $\mathrm{HBB}$ and breast cancer cell invasiveness was demonstrated in human breast cancer cell lines, and overexpression of $\mathrm{HBB}$ enhanced their malignancy, increasing invasion, migration and MMP-9 expression compared to control cells. HBB-induced increase in invasiveness seems to be specific for the more aggressive cell lines. Intriguingly, MCF7 cells presented with upregulation of the epithelial to mesenchymal transition (EMT) markers Vimentin and $\alpha-S M A$, which was reverted by knocking down HBB in MCF7-HBB cells, suggesting that the EMT caused by $\mathrm{HBB}$ is not permanent.

Notably, despite no differences in in vitro cell growth, in vivo primary tumour surrogates of HBB-overexpressing MDA-MB-231 cells were larger than control tumours, relapsing both locally and in distant organs. This apparent contradiction may be explained by the fact that, at variance with an in vitro condition, tumour microenvironment could be influenced by $\mathrm{HBB}$-overexpressing cells at multiple levels. In fact, MDA-HBB tumours were more vascularised and displayed less fibrosis then control tumours. Increased vascularisation may supply more nutrients and mitogens, which could contribute to a faster tumour growth, while the real prognostic meaning of fibrosis in tumours is still unclear (Lunevicious et al, 2001; Radisky and Radisky, 2007; Radisky et al, 2007). Abundant stroma inside the tumour, and between the tumour and its capsule, is associated with a less aggressive phenotype. This assumption seems to be confirmed in our study, in which we found less fibrosis in tumours that were more aggressive.

Interestingly, although prostate cancers share some features with breast cancers, including hormone sensitivity and preferential bone metastatisation (Risbridger et al, 2010), human prostate carcinomas were virtually negative for $\mathrm{HBB}$, except for one single sample over 22 analysed. Despite the undetectable HBB protein in the majority of our prostate cancer samples, bioinformatics tools identified $\mathrm{HBB}$ transcriptional differences in public microarray databases that allowed the stratification of prostate cancer patients in 'higher' and 'lower' HBB groups. In support of our observations, the Kaplan-Meier survival analysis failed to unveil any relevance of these differences on the patient overall survival, confirming that
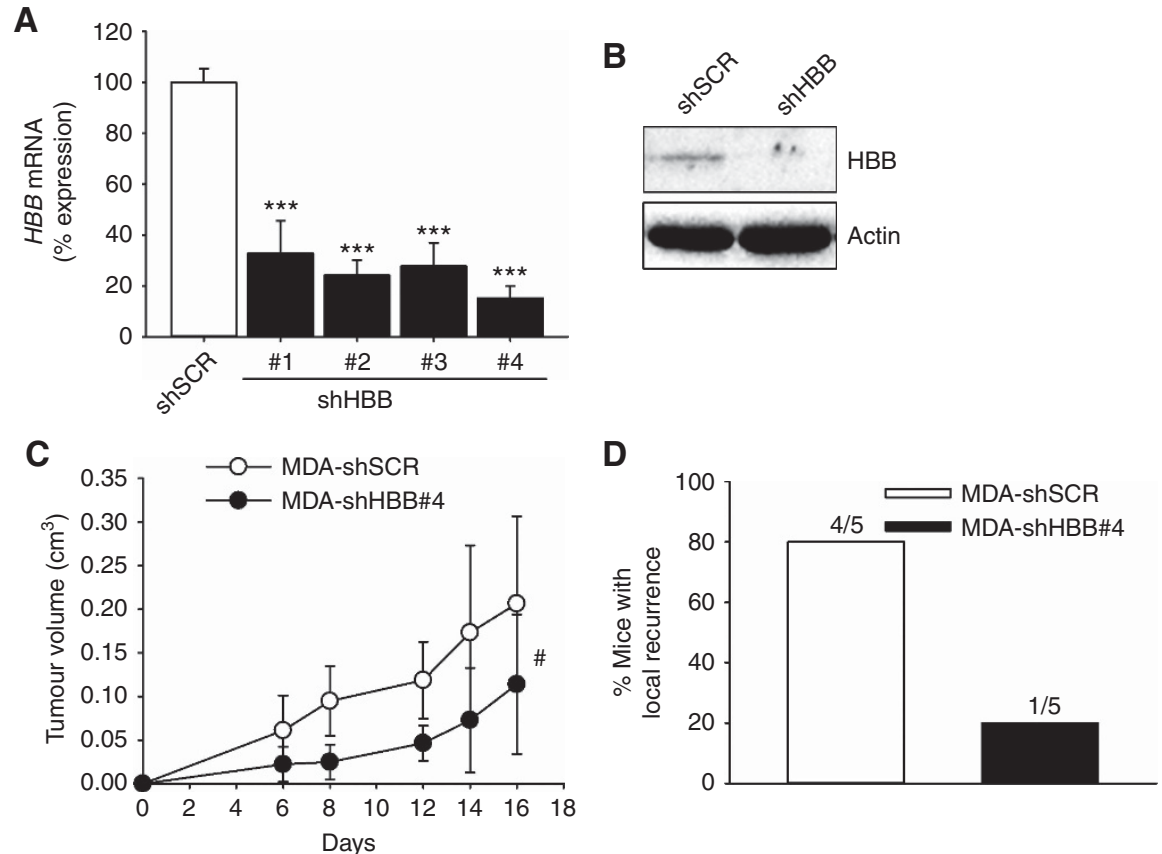

Figure 7. Effect of HBB knock down on in vivo tumour growth. (A) Transcriptional expression of HBB in MDA-MB-231 cells stably transfected with N. 4 small hairpin siRNAs specific for HBB (siHBB\#1, \#2, \#3, \#4) or with scrambled shRNA (shSCR). (B) Evaluation of HBB protein expression in MDA-shSCR and MDA-shHBB protein lysastes (Actin = housekeeping protein). (C, D) Four week-old Balb/cnu/nu female mice were subjected to injection of MDA stable transfected with shRNA\#4 specific for HBB (MDA-shHBB\#4) or with MDA-shSCR $(1 \times 106$ per $50 \mu$ l PBS) into the mammary fat pad. (C) Tumour volume was recorded during the timeframe of the experiment. (D) Percentage of mice with local recurrence evaluated 23 days after tumour resection. Data are the mean \pm s.d. of $n=5$ mice per group ( ${ }^{\#} P=0.04$ vs MDA-shSCR, area under the curve; $\left.{ }^{* \star \star} P<0.002\right)$. 
HBB could have a biological role only in specific cancers, including the invasive breast carcinomas investigated in this study.

With regard to the functional meaning of HBB in breast cancer, one could speculate that it is correlated with the demonstrated ability of heme-proteins to bind not only $\mathrm{O}_{2}$ but also other small molecules with an oxygen atom, such as ROS. Cancer cells produce more ROS than normal cells (Szatrowski and Nathan, 1991). This can lead to an activation of many classical hallmarks of cancer (genomic instability, proliferation, survival, inflammation and angiogenesis) (Irani et al, 1997). On the other hand, there is an emerging evidence that tumour cells also need to fight oxidative damage in order to survive and, most importantly, to metastasize (Hussain et al, 2003). In fact, a large body of evidence indicates that oxidants may also exert tumour-inhibitory actions (Pani et al, 2010). As an example, some chemotherapeutics like doxorubicin, act, at least in part, through ROS generation, while 2-methoxyestradiol was specifically selected for its pro-oxidant capacity (Trachootham et al, 2009). All these data indicate that tumour cells need to find the right balance in ROS presence and activity to favour proliferation and migration, while limiting the risks of antioncogenic responses, such as apoptosis. Therefore, the expression of HBB could be used by tumour cells to prevent the oxidants from becoming too active, as we also showed in our previous work (Capulli et al, 2012). The involvement of HIF-1 $\alpha$, a well-described tumourigenic factor in many types of malignancies (Hirota and Semenza, 2006; Wong et al, 2011; Tiburcio et al, 2014), opens another door over the wide world of oxygen handling: tumour cells are reacting against hypoxia, even though they have plenty of available oxygen. This condition is well portrayed by the term 'oxygen positive hypoxia' (Supplementary Figure 12). We know that intracellular oxygen is provided by free diffusion through membranes, but the presence of intracellular HBB could affect the intracellular $\mathrm{pO}_{2}$ by binding oxygen, thus rendering it unavailable for the cell sensors. This would activate the HIF- $1 \alpha$ pathway, increasing angiogenesis induction and migration (Hirota and Semenza, 2006). This idea is corroborated by the CORM-2 and chetomin experiments, in which blocking the $\mathrm{O}_{2}$ binding site of heme (with CORM-2) or HIF- $1 \alpha$ (with chetomin) in HBB overexpressing cells, obliterates the motility increase induced by HBB overexpression.

Intriguingly, a recent report has shown that microenvironmentderived $\mathrm{HBB}$ can hinder cancer metastatisation of neuroblastoma to lung (Maman et al, 2017). The study showed that the sources of HBB in lung are pneumocytes and endothelial cells rather than the metastatic cancer cells themselves. This environmental HBB could influence tumour engraftment by molecular pathways unrelated to those observed in our study, thus reconciling this apparent discrepancy.

In conclusion, in this work we demonstrated that HBB is expressed by breast cancer cells, its expression is correlated with tumour aggressiveness in humans and its forced overexpression enhances breast cancer cell aggressiveness in vitro and in vivo. We believe that $\mathrm{HBB}$ could be a prognostic marker for breast cancer patients. A systematic study of its expression in breast carcinomas and the analysis of the correlation of this parameter with pathological events, such as relapse and recurrence, could be of great interest for the scientific and oncologic community, with a direct impact on patient lives by rendering their diagnosis more accurate, and therefore their therapy more targeted.

\section{ACKNOWLEDGEMENTS}

This work was supported by grants from the 'Associazione Italiana per la Ricerca sul Cancro' (AIRC) to NR and AT. We are indebted with Dr Rita Di Massimo for her contribution in editing this manuscript and with Gianfranco Ciccone and Anna Rita Nardecchia for their help in animal care.
CONFLICT OF INTEREST

The authors declare no conflict of interest.

\section{REFERENCES}

Aguirre-Gamboa R, Gomez-Rueda H, Martinez-Ledesma E, Martinez-Torteya, Chacolla-Huaringa R, Rodriguez-Barrientos A, Tamez-Peña JG, Treviño V (2013) SurvExpress: an online biomarker validation tool and database for cancer gene expression data using survival analysis. PLoS One 8: 1-9.

Albini A, Iwamoto Y, Kleinman HK, Martin GR, Aarson SA, Kozlowski JM, McEwan RN (2004) A rapid in vitro assay for quantitating the invasive potential of tumour cells. Cancer Res 47: 3239-3245.

Bhaskaran M, Chen H, Chen Z, Liu L (2005) Hemoglobin is expressed in alveolar epithelial type II cells. Biochem Biophys Res Commun 333: 1348-1352.

Bonaventura C, Henkens R, Alayash AI, Banerjee S, Crumbliss AL (2013) Molecular controls of the oxygenation and redox reactions of haemoglobin. Antioxid Redox Signal 18: 2298-2313.

Braden AM, Stankowski RV, Engel JM, Onitilo AA (2013) Breast Cancer Biomarkers: risk assessment, diagnosis, prognosis, prediction of treatment efficacy and toxicity, and recurrence. Curr Pharm Des 20: 4879-4898.

Capulli M, Angelucci A, Driouch K, Garcia T, Clement-Lacroix P, Martella F, Ventura L, Bologna M, Flamini S, Moreschini O, Lidereau R, Ricevuto E, Muraca M, Teti A, Rucci N (2012) Increased expression of a set of genes enriched in oxygen binding function discloses a predisposition of breast cancer bone metastases to generate metastasis spread in multiple organs. J Bone Miner Res 27: 2387-2398.

Chiarugi V, Magnelli L, Chiarugi A, Gallo O (1999) Hypoxia induces pivotal tumor angiogenesis control factors including p53, vascular endothelial growth factor and NF-kappaB-dependent inducible nitric oxide synthase and cyclooxygenase 2. J Cancer Res Clin Oncol 125: 525-528.

Courtnay R, Ngo DC, Malik N, Ververis K, Tortorella SM, Karagiannis TC (2015) Cancer metabolism and the Warburg effect: role of HIF-1 and PI3K. Mol Biol Rep 42: 841-851.

Franceschini A, Szklarczyk D, Frankild S, Kuhn M, Simonovic M, Roth A, Lin J, Minguez P, Bork P, von Mering C, Jensen LJ (2013) STRING v9.1: protein-protein interaction networks, with increased coverage and integration. Nucleic Acid Res 41: D808-D815.

Gorr TA, Wichmann D, Pilarsky C, Theurillat JP, Fabrizius A, Laufs T, Bauer T, Koslowski M, Horn S, Burmester T, Hankeln T, Kristiansen G (2011) Old proteins-new locations: myoglobin, haemoglobin, neuroglobin and cytoglobin in solid tumours and cancer cells. Acta Physiol 202: 563-581.

Hirota K, Semenza GL (2006) Regulation of angiogenesis by hypoxia-inducible factor 1. Crit Rev Oncol Hematol 59: 15-26.

Hussain SP, Hofseth LJ, Harris CC (2003) Radical causes of cancer. Nat Rev Cancer 3: 276-285.

Irani K, Xia Y, Zweier JL, Sollott SJ, Der CJ, Fearon ER, Sundaresan M, Finkel T, Goldschmidt-Clermont PJ (1997) Mitogenic signaling mediated by oxidants in Ras-transformed fibroblasts. Science 275: 1649-1652.

Liu L, Zeng M, Stamler JS (1999) Hemoglobin induction in mouse macrophages. Proc Natl Acad Sci USA 96: 6643-6647.

Lu YS, Yen PI, Chuang SE, Gao M, Kuo ML, Cheng AL (2006) Glucocorticoids enhance cytotoxicity of cisplatin via suppression of NFkappaB activation in the glucocorticoid receptor-rich human cervical carcinoma cell line SiHA. J Endocrinol 188: 311-319.

Lunevicious R, Nakanishi H, Ito S, Kozaki K, Kato T, Tatematsu M, Yasui K (2001) Clinicopathological significance of fibrotic capsulae formation around liver metastases from colorectal cancer. J Cancer Res Clin Oncol 127: 193-199.

Maman S, Sagi-Assif O, Yuan W, Ginat R, Meshel T, Zubrilov I, Keisari Y, Lu W, Lu W, Witz IP (2017) The beta subunit of hemoglobin (HBB2/HBB) suppresses neuroblastoma growth and metastasis. Can Res 77: 14-26.

Nishi H, Inagi R, Kato H, Tanemoto M, Kojima I, Son D, Fujita T, Nangaku M (2008) Hemoglobin is expressed by mesangial cells and reduces oxidant stress. J Am Soc Nephrol 19: 1500-1508.

Pani G, Galeotti T, Chiarugi P (2010) Metastasis: cancer cell's escape from oxidative stress. Cancer Metastasis Rev 29: 351-378.

Radisky DC, Kenny PA, Bissell MJ (2007) Fibrosis and cancer: do myofibroblasts come also from epithelial cells via EMT? J Biol Chem 101: 830-839. 
Radisky ES, Radisky DC (2007) Stromal induction of breast cancer: inflammation and invasion. Rev Endocr Metab Disord 8: 279-287.

Reed E, Kössler I, Hawthorn J (2012) Quality of life assessments in advanced breast cancer: should there be more consistency? Eur J Cancer Care 21: $565-580$.

Risbridger GP, Davis ID, Birrell SN, Tilley WD (2010) Breast and prostate cancer: more similar than different. Nat Rev Cancer 10: 205-212.

Rucci N, Capulli M, Ventura L, Angelucci A, Peruzzi B, Tillgren V, Muraca M, Heinegard D, Teti A (2013) Proline/arginine-rich end leucine-rich repeat protein $\mathrm{N}$-terminus is a novel osteoclast antagonist that counteracts bone loss. J Bone Miner Res 28: 1912-1924.

Rucci N, Ricevuto E, Ficorella C, Longo M, Perez M, Di Giacinto C, FunariA, Teti A, Migliaccio N (2004) In vivo bone metastases, osteoclastogenic ability and phenotypic characterization of human breast cancer cells. Bone 34: 697-709.

Suzuki Y, Takeda Y, Ikuta T (2008) Immunoblotting for human hemoglobin chains. Anal Biochem 378: 218-220.

Szatrowski TP, Nathan CF (1991) Production of large amounts of hydrogen peroxide by human tumour cells. Cancer Res 51: 794-798.
Tiburcio PD, Choi H, Huang LE (2014) Complex role of HIF in cancer: the known, the unknown, and the unexpected. Hypoxia 42: 59-70.

Trachootham D, Alexandre J, Huang P (2009) Targeting cancer cells by ROS-mediated mechanisms: a radical therapeutic approach? Nat Rev Drug Discov 8: 579-591.

Vernon RB, Sage EH (1995) Between molecules and morphology. Extracellular matrix and creation of vascular form. Am J Pathol 147: 873-883.

Wong CC, Gilkes DM, Zhang H, Chen J, Wei H, Chaturvedi P, Fraley SI, Wong CM, Khoo US, Ng IO, Wirtz D, Semenza GL (2011) Hypoxiainducible factor 1 is a master regulator of breast cancer metastatic niche formation. Proc Natl Acad Sci USA 108: 16369-16374.

This work is published under the standard license to publish agreement. After 12 months the work will become freely available and the license terms will switch to a Creative Commons AttributionNonCommercial-Share Alike 4.0 Unported License.

Supplementary Information accompanies this paper on British Journal of Cancer website (http://www.nature.com/bjc) 